\title{
INTERSTELLAR EXTINCTION OF PLANETARY NEBULAE
}

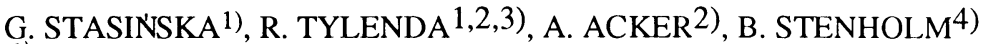 \\ 1) DAEC, Observatoire de Paris-Meudon, France \\ 2) Observatoire de Strasbourg, France \\ 3) Copernicus Astronomical Center, Torun, Poland \\ 4) Lund Observatory, Sweden
}

We have compared the extinction of planetary nebulae (PN) as derived from the Balmer decrement and from the ratio of radio to $\mathrm{HB}$ fluxes.

From a compilation of observational data we have selected PN for which the $6 \mathrm{~cm}(5 \mathrm{GHz})$ radio flux has been measured with the VLA and for which reliable measurements of the H $\beta$ flux and the $\mathrm{H} \alpha / \mathrm{H} \beta$ ratio are available. After a careful discussion we have rejected objects having a 6 $\mathrm{cm}$ flux below $10 \mathrm{mJy}$ or a $6 \mathrm{~cm}$ brightness temperature below $10 \mathrm{~K}$, as their radio flux measurements are presumably less accurate. In this way we have obtained a sample of $\sim 130 \mathrm{PN}$ for which we have derived the extinction constants both from the $\mathrm{H} \alpha / \mathrm{H} \beta$ ratio $\left(\mathrm{C}_{\mathrm{opt}}\right)$ and from the radio to $\mathrm{H} \beta$ flux ratio $\left(\mathrm{C}_{\mathrm{rad}}\right)$. The results are compared in the figure below which shows that $\mathrm{C}_{\mathrm{Opt}}$ is systematically larger than $\mathrm{C}_{\mathrm{rad}}$. The orthogonal regression line (dashed in the figure) is: $\mathrm{C}_{\text {opt }}=(1.19 \pm 0.03) \mathrm{C}_{\mathrm{rad}}+(-0.01 \pm 0.03)$.

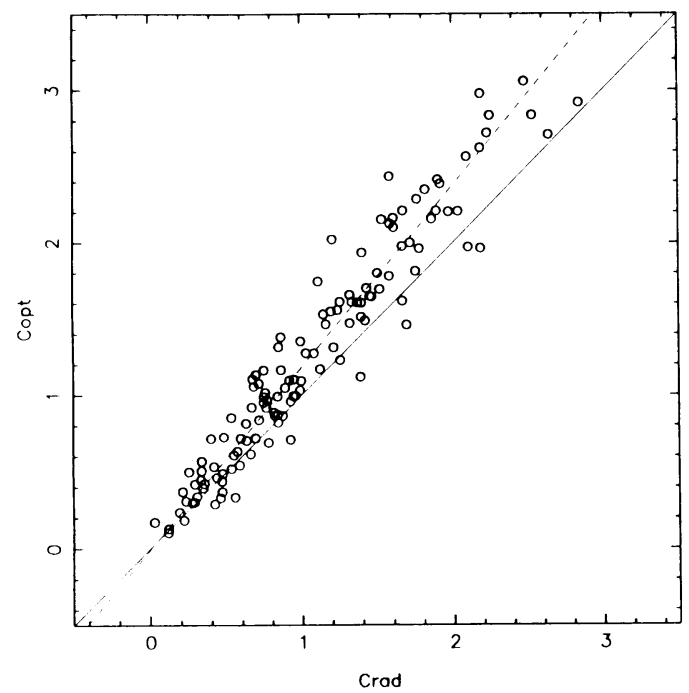

This result is opposite to the well known effect observed in extragalactic HII regions which is generally explained by the presence of large amounts of local dust (inside and/or surrounding the HII region). We argue that the effect we have found for the PN cannot be entirely attributed to systematic observational errors although the problem of nonlinearity of some optical detectors needs to be clarified. Circumnebular dust, although undoubtfully present in some - especially young - PN, cannot be responsible for the bulk of the extinction observed for most of the PN. Instead, we suggest that, for most of distant PN, the total to selective extinction, $\mathrm{RV}$, is significantly lower than 3 , the value usually adopted for the standard interstellar extinction law.

A detailed report on the subject is in press in Astronomy and Astrophysics. 